Tabelle 1: Ergebnisse (in \%) zur Frage nach der anzustrebenden Ausbildungsart. Daten dargestellt als Prozentsatz in Bezug auf die Gesamtteilnehmer (Gesamt \%), des wissenschaftlichen Nachwuchses (WN \%) und der etablienten Wissenschaftler (EW \%)

\begin{tabular}{l|c|c|c}
\hline Ausbildungsart & Gesamt \% & WN \% & EW \% \\
\hline Postgraduale Zertifizierung & 25,6 & 17,5 & 32,6 \\
\hline Integration in NaWi-Studieng. & 57,0 & 62,5 & 52,2 \\
\hline Grundständiger Studiengang & 17,4 & 20,0 & 15,2 \\
\hline
\end{tabular}

ge aufgeworfen, wie und von wem eine solche Zusatzqualifikation finanziert werden solle und zu bedenken gegeben, dass die Studenten immer älter werden würden, bis sie als 'gut ausgebildet' gelten und damit im internationalen Vergleich benachteiligt wären. Auch wurde angemahnt, dass besonders Frauen von einer solchen Zusatzausbildung nach dem Studium hinsichtlich der Lebens-/Familienplanung diskriminiert würden, da diese durch den Zeitverlust durch Schwangerschaft, Erziehungsurlaub und auch noch einer Weiterqualifikation ihre Chancengleichheit auf dem Arbeitsmarkt gefährdet wäre.

Insgesamt muss aber berücksichtigt werden, dass eine gute Ausbildung auch finanziert werden muss, z.B. durch die Anstellung junger Wissenschaftler in Drittmittelprojekten. Daher wurde eine verbesserte Forschungs- und Ausbildungsförderung als höchste Priorität (26,7\%) bei der Verbesserung im Berufsfeld Ökotoxikologie gesehen, gefolgt von einer verbesserten Ausbildung, Netzwerkbildung und Kommunikation. Gerade in Hinblick der zurückgeschraubten Förderung durch Drittelmittelgeber unterstreicht die Forderung nach einer verbesserten Ausbildungsfinanzierung in der Ökotoxikologie das Problem, das es nur gut ausgebildete Ökotoxikologen geben kann, wenn genug Gelder für Drittmittelprojekte zur Verfügung stehen und somit junge Wissenschaftler in die Forschung mit eingebunden werden können. Dies steht im deutlichen Widerspruch zur derzeitigen ökotoxikologischen Projektförderung, die bei allen großen Forschungsträgern in den letzten Jahren starke Einschnitte erfahren hat (vgl. Sturm 2003).

Eine verbesserte Integration der Auszubildenden (Diplomanden und Doktoranden) in die laufende Forschung war somit eine weitere Forderung des wissenschaftlichen Nachwuchses. Auch wenn während der SETAC-Tagung von Vertretern der Behörden, die Notwendigkeit gut ausgebildeter Ökotoxikologen in Deutschland formuliert wurde, war die Mehrheit der Befragten in diesem Punkt sehr skeptisch. Während 50,7\% den Bedarf an Ökotoxikologen als mittelmäßig einschätzen, bewerteten $27,5 \%$, den Bedarf als eher gering bzw. sehr gering $(11,6 \%)$. Daher glauben auch nur $17,7 \%$ aller Befragten, dass die Berufschancen für den wissenschaftlichen Nachwuchs (längerfristig) in der Ökotoxikologie gut sind, während $51,5 \%$ sie für mittelmäßig und 29,4\% sie für schlecht ansehen.

Diese Diskrepanz zeigt deutlich, dass auch hier die Kommunikation zwischen den Entscheidungsträgern (Behörden), die mehr gut ausgebildete Ökotoxikologen einfordern, und den Wissenschaftlern, die befürchten, dass nicht genügend langfristige Arbeitsplätze für
Ökotoxikologen zur Verfügung stehen, verbessert werden muss. Zum anderen sollte ein Abgleich des 'Angebotes (gut ausgebildete Ökotoxikologen) und der Nachfrage (Anzahl der Arbeitsplätze)' erfolgen. Dies ist gleichzusetzen mit der Forderung des wissenschaftlichen Nachwuchses nach mehr Transparenz in der Ausbildungspolitik, neben einer verbesserten Integration der Nachwuchswissenschaftler nicht nur in der Forschung, sondern auch in der Diskussion über die Ausbildung in der Ökotoxikologie. Nach Meinung der SETAC-GLB Initiative ist hierzu ein Umdenken in dem deutschen hierarchisch aufgebauten Wissenschaftswesen notwendig. Einen ersten wichtigen Impuls in diese Richtung hat hier die SETAC mit ihrer Tagung der SETAC-GLB in Heidelberg gegeben, die für den wissenschaftlichen Nachwuchs ein ausgezeichnetes Forum bot. Junge Wissenschaftler konnten zum einen ihre Forschungsergebnisse in Form von Postern und Vorträgen vorstellen und zum anderen auch die Diskussion mit den etablierten Wissenschaftlern suchen. Weitere Schritte in diese Richtung müssen folgen. Um den Bedarf an gut ausgebildeten Ökotoxikologen zu decken, sollten

- ökotoxikologische Lerninhalte schon in einem frühen Stadium des Studiums vermittelt werden (nicht erst während oder nach der Promotion, wie es z.B. ein Postgradualstudium mit zertifizierten Abschluss als Fachökotoxikologe vorsehen würde),

- die finanzielle Förderung der Ausbildung gesichert werden,

- die Förderungen von ökotoxikologischen Drittmittelprojekten (Promotionsstellen!) durch Projektträger, die in den letzten Jahren starke Einschnitte erfahren hat, wieder ausgebaut werden und

- die Nachwuchswissenschaftler in die Diskussion um die Ausbildung der Ökotoxikologen einbezogen werden.

Folglich begrüßt der wissenschaftliche Nachwuchs, dass eine Diskussion zur Ausbildung in der Ökotoxikologie in Gang gekommen ist, die Stellung der Nachwuchswissenschaftler im Wissenschaftssystem neu beleuchtet wird und sie in Entscheidungsprozessen hinsichtlich der Ausbildung mit integriert werden sollen.

Daher gilt der SETAC-GLB und speziell den Organisatoren der Jahrestagung 2003 in Heidelberg einen besonderen Dank, die den Wissenschaftlichen Nachwuchs unterstützt und die Gelegenheit gegeben haben, sich für ihre Anliegen Gehör zu schaffen. Besonderen Dank geht hierbei an Henner Hollert, der die SETAC-GLB Initiative sowohl zu ihrer Fragebogenaktion als auch bei ihrer Idee eine Podiumsdiskussion zu veranstalten bestärkt und unterstützt hat.

\section{Literatur}

Ahlers J, Filser J, Frank H, Gies A, Klein W, Nagel R, Schüürmann G (2003) Editorial: Ökotoxikologie soll endlich wissenschaftliches Fach werden. UWSF - Z Umweltchem Ökotox 15 (1) 3-4

Hollert H, Braunbeck T (2003): SETAC Europe-GLB Corner: Nachwuchsförderung in der Ökotoxíkologie - New Blood in Ecotoxicology, Achte Deutschsprachige Jahrestagung der SETAC Europe-GLB, UWSF - Z Umweltchem Ökotox 15 (4) $281-282$

Sturm K-D (2003): Leserbrief zur Berliner Erklärung zur Ökotoxikologie und Ökotoxikologenausbildung. UWSF - Z Umweitchem Ökotox 15 (2) 68

\title{
Nachwuchs-Förderpreis der SETAC-GLB
}

Der Vorstand der SETAC-GLB wird im Jahr 2004 erstmalig einen Förderpreis für NachwuchswissenschaftlerInnen auf der Tagung in Aachen verleihen, insbesondere, um auf die Notwendigkeit einer Verbesserung der Ausbildungssituation und der Forschungsförderung in der Umweltforschung aufmerksam zu machen.

Es werden wissenschaftliche Arbeiten (z.B.: Diplom, Magister, Dissertation o.ä.) aus den Themengebieten der SETAC (http://www.setac-glb.de) im weitesten Sinne erwartet. Die Themengebiete können sich über folgende Bereiche erstrecken:

Biologie, Umweltchemie, Anatomie/Physiologie, Naturschutz, Klassische Toxikologie, Ökologie, Ökotoxikologie, Genetik, Umweltrecht, Mikrobiologie, Organische Chemie, Analytische Chemie und Untersuchung der Umweltsphären Boden, Wasser und Luft.

Die eingereichte Arbeit soll nicht länger als drei Jahre zurückliegen. Die Bewertung wird durch ein vom Vorstand bestimmtes Gremium durchgeführt. Die von der Jury vorgeschlagene Arbeit wird auf der SETAC-GLB
Tagung in Aachen 2004 mit einem Ausbildungsförderpreis im Wert von 3000,00 Euro gekürt.

Die Arbeiten sind bei der Geschäftsstelle der SETAC-GLB einzureichen Die Auszahlung des Preisgeldes wird durch den Bewerber beim Vorstand beantragt. Ein Verwendungsnachweis im Sinne der Aus- oder Fortbildung ist zu führen. Hierzu zählen z.B. Ausbildungsgebühren von laufenden oder geplanten Projekten sowie der Besuch von Fachtagungen

Abgabetermin: 31. Mai 2004

Einzureichende Unterlagen in deutsch oder englisch bitte jeweils als pdf-file:

- Die wissenschaftliche Arbeit

- Kurzfassung der Arbeit (max. eine Seite)

- Lebenslauf

- Gewünschte Verwendung des Preisgeldes (kurze Beschreibung des Projektes und Einbindung in die eigene Ausbildung)

Die Unterlagen/Rückfragen bitte an folgende Mailadresse:

z. Hd. Dr. Peter Ebke, email: award@ setac-glb.org 